\title{
General relativistic magnetohydrodynamic simulations of collapsars: Rotating black hole $\operatorname{cases}\left({ }^{*}\right)$
}

\author{
Y. $\operatorname{Mizuno}\left({ }^{1}\right)$, S. $\operatorname{Yamada}\left({ }^{2}\right)$, S. $\operatorname{Koide}\left({ }^{3}\right)$ and K. Shibata $\left({ }^{4}\right)$ \\ $\left({ }^{1}\right)$ Department of Astronomy, Kyoto University - Kyoto, Japan \\ $\left({ }^{2}\right)$ Science and Engineering, Waseda University - Tokyo, Japan \\ $\left(^{3}\right)$ Department of Engineering, Toyama University - Toyama, Japan \\ $\left({ }^{4}\right)$ Kwasan and Hida Observatory, Kyoto University - Kyoto, Japan
}

(ricevuto il 23 Maggio 2005; pubblicato online il 22 Settembre 2005)

\begin{abstract}
Summary. - We have performed 2.5-dimensional general relativistic magnetohydrodynamic (MHD) simulations of collapsars including a rotating black hole. Initially, we assume that the core collapse has failed in this star. A rotating black hole of a few solar masses is inserted by hand into the calculation. The simulation results show the formation of a disklike structure and the generation of a jetlike outflow near the central black hole. The jetlike outflow propagates and accelerated mainly by the magnetic field. The total jet velocity is $\sim 0.3 c$. When the rotation of the black hole is faster, the magnetic field is twisted strongly owing to the frame-dragging effect. The magnetic energy stored by the twisting magnetic field is directly converted to kinetic energy of the jet rather than propagating as an Alfvén wave. Thus, as the rotation of the black hole becomes faster, the poloidal velocity of the jet becomes faster.
\end{abstract}

PACS 98.70.Rz - $\gamma$-ray sources; $\gamma$-ray bursts.

PACS 95.30.Qd - Magnetohydrodynamics and plasmas.

PACS 95.30.Sf - Relativity and gravitation.

PACS 01.30.Cc - Conference proceedings.

\section{1. - Introduction}

It is now generally believed that "long-soft" gamma-ray bursts (GRBs) are a phenomenon related to the deaths of massive stars. There is direct and indirect observational evidence of the close relationship between GRBs and supernovae, such as the observed association with star-forming regions in galaxies [1], the "bump" observed in the afterglows of some GRBs [2, 3], and the association of GRB 980425 with SN 1998bw [4], GRB 030329 with SN 2003dh [5], and GRB031303 with SN 2003lw [6]. It is thus probable that a major subclass of GRBs is a consequence of the collapse of a massive star.

$\left(^{*}\right)$ Paper presented at the "4th Workshop on Gamma-Ray Burst in the Afterglow Era", Rome, October 18-22, 2004.

(C) Società Italiana di Fisica 
The collapsar model is one of the most promising scenarios involving massive stars $[7$, 8]. A collapsar is a rotating massive star that lacks a hydrogen envelope. In this model, the iron core of the rotating massive star collapses to a black hole surrounded by an accretion disk. The accretion through this disk produces outflow via neutrino annihilation and/or magnetohydrodynamic (MHD) processes. They are further collimated by the passage through the stellar mantle. The formation and propagation of relativistic flows from collapsars have been studied numerically by Newtonian hydrodynamic [8, 9], special relativistic hydrodynamic [10, 11, 12], Newtonian MHD [13], and general relativistic MHD simulations [14, 15]

Here, as a collapsar model we perform 2.5-dimensional general relativistic MHD simulations of the gravitational collapse of a rotating magnetized massive star with a rotating black hole at the center.

\section{2. - Numerical method}

In order to study the formation of relativistic jets from collapsar we use a 2.5dimensional general relativistic magnetohydrodynamics (GRMHD) code [16, 14].

As for the initial model, we have the collapsar model in mind. We employ only the profiles of the density, pressure and radial velocity as our initial condition from the postbounce profile of Bruenn's realistic 1D supernova model [17]. We put a few $M_{\odot}$ rotating black hole at the center. We add by hand the stellar rotation and intrinsic magnetic field in the simulations. See Mizuno's recent papers $[14,15]$ for details.

\section{3. - Numerical results}

The simulations were performed corotating cases with different rotation parameter of a black hole. From the snapshots of density and plasma beta (fig. 1) all models show similar results. The stellar matter falls onto the central black hole at first. The matter piles up on the equatorial plane and forms a disklike structure. Since the magnetic field is frozen into the plasma, it is dragged and deformed by the accreting matter. Then a shock wave and jetlike outflow are produced near the central black hole. The jetlike outflow is generated and accelerated mainly by the magnetic field. The jet has a mildly relativistic velocity $\sim 0.3 c$. The total velocity of jet is clearly larger than the velocity of circular orbit of a particle around a black hole (Kepler velocity). This means that the jetlike outflow is likely get out of the stellar remnant.

There are some difference and dependence in these simulations. Figure 2 shows the dependence of the properties of jet on the rotation parameter of the black hole. For smaller values of the rotating parameter, the jet is ejected from more outer regions and the propagation of the amplified magnetic field as Alfvén waves is faster and is seen more clearly. This implies that the inner magnetic field is amplified strongly by the framedragging effect. From the distribution of velocity and the ratio of toroidal to poloidal magnetic field components along the jet in the cases with different rotation parameter of a black hole, as the rotation of a black hole becomes slower, the toroidal component of the velocity becomes slower. The ratio of toroidal to poloidal magnetic field components gets larger gradually as the black hole is faster. These results are understood from how effective the frame-dragging effect is. As the rotation of the black hole is faster, the magnetic energy stored by the twisted magnetic field is converted to kinetic energy of the jet more directly rather than to the propagation of Alfvén waves. As a result, the poloidal velocity of the jet becomes higher. 


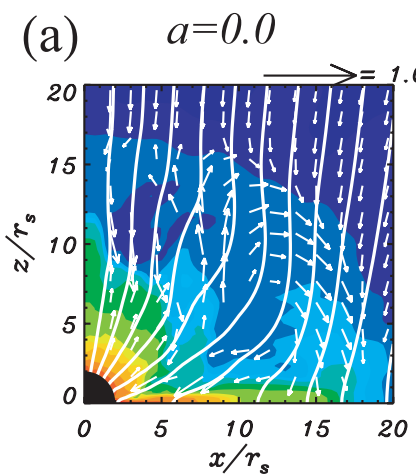

(d)

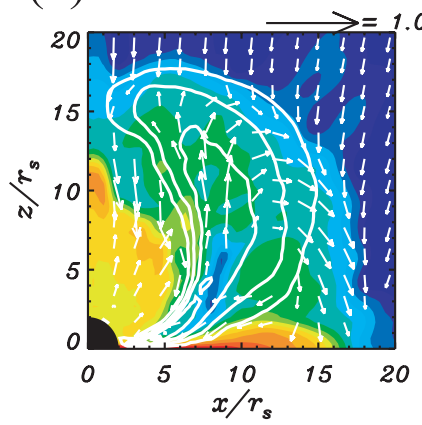

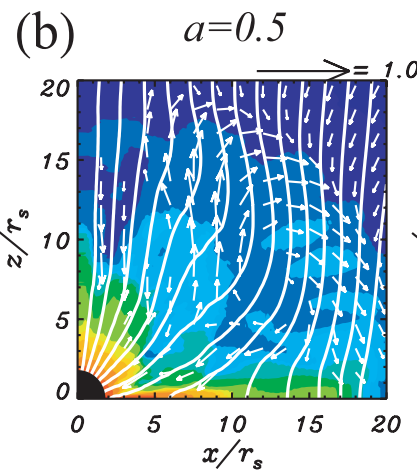

(e)

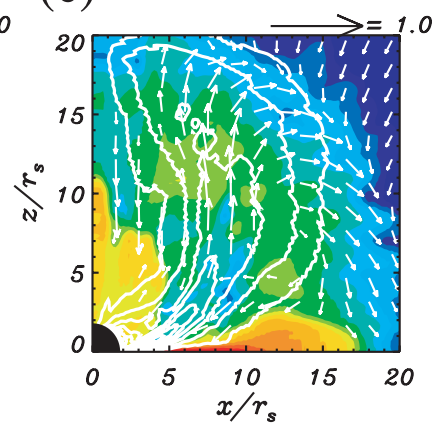

(c) $\quad a=0.9$

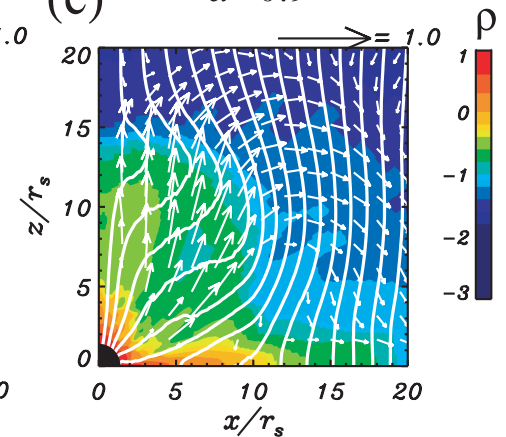

(f)

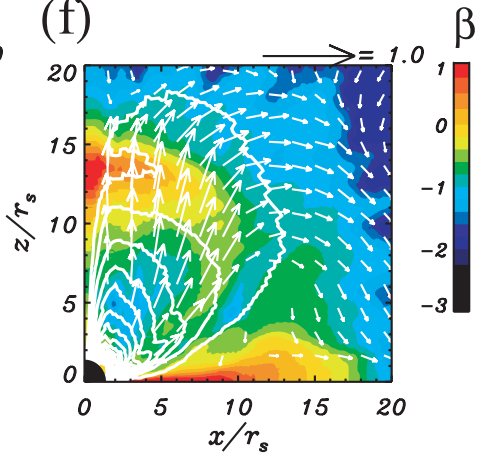

Fig. 1. - Snapshots of density and plasma beta in the corotating cases having different rotation parameter at $t / \tau_{S}=136$. The grey scales show the values of the logarithm of density and plasma beta. The white curves represent $(a-c)$ the magnetic field lines and $(d-f)$ the contour of the toroidal magnetic field. Arrows depict the poloidal velocities normalized by the light-velocity.

We can also see the deference in these simulations from the time variations of the flux of kinematic energy, and Poyniting flux. In the rapidly rotating black hole case $(a=0.999)$, the kinetic energy flux is comparable to the Poynting flux. These results differ from those for the nonrotating black hole case $(a=0.0)$. This is because the jetlike outflow for the rotating black hole is also larger, because the magnetic field is twisted strongly owing to the frame-dragging effect of the rotating black hole.

\section{4. - Discussion}

We discuss the application of our results to the central engine of GRBs. The maximum jet velocity in our simulations including a rotating black hole was about $0.3 c$. The typical kinetic energy of the jet was $\simeq 10^{54}$ ergs assuming the density of the jet was $\simeq 10^{13} \mathrm{~g} / \mathrm{cm}^{3}$. Although it may somewhat overestimate, this is consistent with the standard energy of GRBs. However, the jet is too slow compared to the velocity which has been inferred for the jet of real GRBs. We have to consider other acceleration mechanisms. On the other hand, our results can be applied to baryon-rich outflows associated with failed GRBs. It is a fireball with a high baryonic load and mildly relativistic velocities. SN 2002ap is a candidate for failed GRBs. Although it is not associated with a GRB, it has a jet with a velocity of $0.23 \mathrm{c}$ and estimated kinetic energy of the jet is $\sim 5 \times 10^{50} \mathrm{ergs}[18]$. The jet in our rotating black hole case has enough velocity and energy to apply to the jet model 

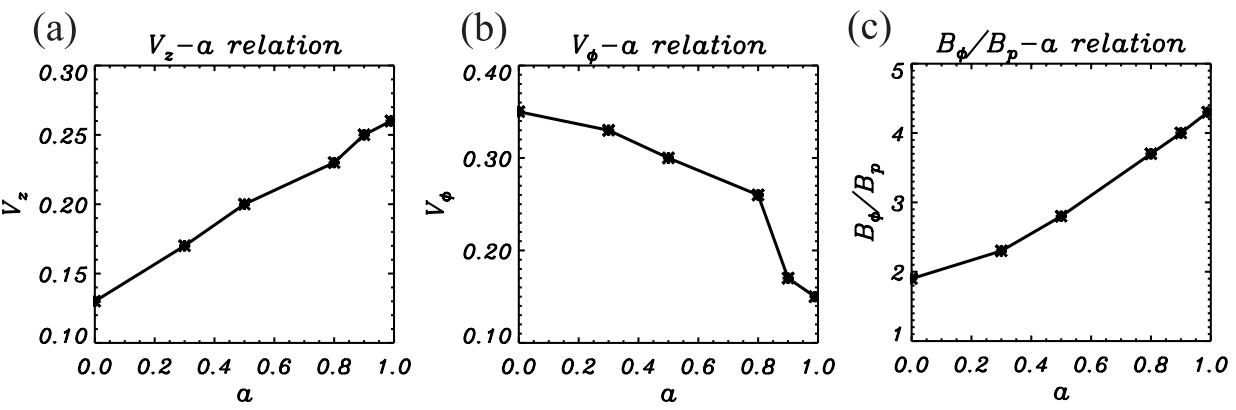

Fig. 2. - Dependence of $(a)$ maximum vertical velocity of the jet $\left(v_{z}\right),(b)$ the maximum toroidal velocity of the jet $\left(v_{\phi}\right)$, and $(c)$ the maximum ratio of toroidal to poloidal magnetic field components $\left(B_{\phi} / B_{p}\right)$ of the jet on the rotation parameter of a black hole, $a$, in the corotaitng case.

of SN2002ap.

In our simulations, we have neglected microphysics (photo-disintegration, treatment of neutrinos, etc.) and concentrated on the general relativistic MHD, particularly the effect of rotating black hole. Such treatment may be reasonable as a first step. However, microphysics are critically important for realistic GRMHD simulations of collapsars.

This work was partially supported by a Grant-in-Aid for the 21st Century COE "Center for Diversity and Universality in Physics".

\section{REFERENCES}

[1] Bloom J. S., Kulkarni S. R. and Djorgovski G., AJ, 123 (2002) 1111.

[2] Galama T. et al., ApJ, 536 (2000) 185.

[3] Bloom J. S. et al., ApJ, 572 (2002) L45.

[4] Galama T. et al., Nature, 395 (1998) 670.

[5] StaneK K. Z. et al., ApJ, 591 (2003) L111.

[6] Malesani D. et al., ApJ, 609 (2004) L5.

[7] Woosley S. E., ApJ, 405 (1993) 273.

[8] MacFadyen A. I. and Woosley S. E., ApJ, 524 (1999) 262.

[9] MacFadyen A. I., Woosley S. E. and Heger A., ApJ, 550 (2001) 410.

[10] Aloy M. A. et al., ApJ, 531 (2000) L119.

[11] Zhang W., Woosley S. E. and MacFadyen A. I., ApJ, 586 (2003) 356.

[12] Zhang W., Woosley S. E. and Heger A., ApJ, 608 (2004) 365.

[13] Proga D., Macfadyen A. I., Armitage P. J. and Begelman M. C., ApJ, 599 (2003) L5.

[14] Mizuno Y., Yamada S., Koide S. and Shibata K., ApJ, 606 (2004) 395.

[15] Mizuno Y., Yamada S., Koide S. and Shibata K., ApJ, 615 (2004) 389.

[16] Koide S., Phys. Rev. D, 67 (2003) 104010.

[17] Bruenn S. W., in Nuclear Physics in the Universe, edited by Guidry M. W. and Strayer M. R. (IOP, Philadelphia) 1992, pp. 31.

[18] Totani T., ApJ, 598 (2003) 1151. 